\title{
Online-Wörterbücher als potenzielles Hilfsmittel beim Sprichwortlernen am Beispiel DaF
}

\author{
SAŠA JAZBEC \\ BRIGITA KACJAN \\ Universität Maribor, Slowenien
}

Received: 6 March 2014 / Accepted: 16 November 2015

ISSN: $1697-7467$

\begin{abstract}
ABSTRAKT: Ausgehend davon, dass Sprichwörter einen natürlichen Teil jeder Sprache darstellen und das Wörterbuch ein unabdingbarer Bestandteil jedes Fremdsprachenunterrichts ist, widmet sich der Beitrag der Frage, ob und in welchem Umfang bestimmte, als frequent ermittelte Sprichwörter in drei exemplarisch ausgewählten elektronischen Wörterbüchern zu finden sind. Darüber hinaus wird untersucht, ob sich mit der festgestellten abnehmenden Frequenz der Sprichwörter auch ihre Präsenz in den untersuchten Wörterbüchern verringert. Mit den exemplarischen Ergebnissen dieser Untersuchung wird ein bedeutendes Desiderat ausgefüllt, denn die Aktualität und Relevanz von Sprichwörtern für Fremdsprachenlerner wurden bisher in der Fachliteratur nicht systematisch untersucht. Abschließend werden ganz kurz einige parömiodidaktische Richtlinien angerissen, die eine bessere Präsenz von Sprichwörtern im Medium Wörterbuch gewährleisten und somit den Fremdsprachenunterricht positiv beeinflussen könnten.

Schlüsselwörter: Online-Wörterbücher, Sprichwort, Deutsch als Fremdsprache, Projekt SprichWort.
\end{abstract}

Online Dictionaries as a potential Proverb Learning Tool: A Case Study of Teaching German as a Foreign Language

\begin{abstract}
Since proverbs constitute a natural part of every language and dictionaries are indispensable medial aids in foreign language teaching, the article addresses the question of whether and to what extent proverbs are present in three different types of dictionaries. Furthermore, this study examines whether the observed decreasing frequency of proverbs is reflected in a decreasing presence of proverbs in these dictionaries. With the results of this survey, an important desideratum is filled, since the topicality and relevance of proverbs for students of foreign languages have not yet been systematically studied in the literature. Ultimately, some paremio-didactic guidelines will be briefly outlined that aim at an improved presence of proverbs in dictionaries and should thus favourably affect foreign language teaching.
\end{abstract}

Keywords: online dictionaries, proverbs, German as a foreign language, project SprichWort.

\section{EINFÜHRUNG}

Was nicht ist, kann ja noch werden ist linguistisch betrachtet ein Sprichwort, das dem Titel vorausgeschickt werden könnte und in diesem Fall mindestens zweideutig verstanden werden müsste: Auf der einen Seite würde es einen der zwei Untersuchungsgegenstände, also 
Sprichwörter, ankündigen, und auf der anderen Seite würde es die Resultate der Untersuchung vorwegnehmen, womit das phraseodidaktische und parömiologisch-lexikographische Forschungsfeld (Kispal, 2007:414) mit einem noch nicht untersuchten Segments bereichert würde.

Der Ausgangspunkt der Untersuchung war folgende in verschiedenen Forschungsarbeiten erkennbare Dichotomie: Einerseits kann man sich die Sprache nicht ohne phraseologische Einheiten, wozu auch die Sprichwörter gerechnet werden, vorstellen, andererseits werden sie im fremdsprachlichen Unterricht auf den unterschiedlichsten Niveaus (in den Lehrwerken, im Gemeinsamen europäischen Referenzrahmen für Sprachen (Europarat, 2001), in Tests usw.) ausgeblendet oder man findet sie nur auf den beiden höchsten Niveaus der Sprachbeherrschung (C1 und $\mathrm{C} 2$ nach dem Referenzrahmen).

Im Beitrag wird im Kontext des Fremdsprachenlernens, speziell des DaF-Lernens, der Frage nachgegangen, ob und welche Sprichwörter in elektronischen Wörterbüchern vorhanden und wie stark sie dort jeweils vertreten sind. Für die Untersuchung wurden illustrativ drei lexikographisch gesehen verschiedene Typen elektronischer Wörterbücher ausgewählt: ein einsprachiges allgemeines Wörterbuch (Das digitale Wörterbuch der deutschen Sprache $(D W D S)$ ), ein einsprachiges Lernerwörterbuch (Langenscheidt) und ein zweisprachiges deutschslowenisches Wörterbuch (PONS). Den Resultaten der empirischen Analyse und Diskussion der Ergebnisse wurden theoretische Ausführungen zum Untersuchungsgegenstand, zu Sprichwörtern und Wörterbüchern, einem wichtigen Medium im Fremdsprachenunterricht, vorausgeschickt.

\section{SPRICHWÖRTER ALS SPRACHLICHES PHÄNOMEN}

Das Sprichwort, ein auf den ersten Blick kleines und nicht wesentliches Element der Sprache, entpuppt sich bei genauerem Studium als komplex und kompliziert, aber auch als kaum endgültig definierbar. Alle Begriffsauffassungen, und davon gibt es viele (vgl. z. B. Wiegand et. al., 1989; Mieder, 2010; Steyer, 2012), kann man sich bildlich wie ein dichtes Netz vorstellen, das das Sprichwort bzw. die Sprichwörter auffangen will. Aber durch die Maschen des Netzes schlüpft immer wieder das eine oder andere Sprichwort raus, aber auch rein. Anders gesagt: Sprichwörter sind eine flexible, sogar amorphe Substanz, eigentlich vorgeformte Formeln, die sich trotz ihrer Festigkeit der Zeit und den Umständen des Gebrauchs anpassen.

Jede linguistische Untersuchung, die sich mit Sprichwörtern auseinandersetzt, fängt im Grunde mit einer theoretischen Auslegung des Begriffs Sprichwort an: entweder hinsichtlich ihres Status im Gesamtsystem der Sprache, im Rahmen der Idiomatik, ihres Funktionierens in der heutigen Kommunikation (Ďurčo, 2005:9) oder mit Bezug auf die Veränderungsprozesse (Ulčnik, 2011). Darin werden die linguistischen Merkmale von Sprichwörtern untersucht, wie etwa Form, Funktion, Pragmatik, Semantik, oder es wird auf die volkskundlichen Merkmale aus den Bereichen Etymologie oder Migration, oder auf kulturelle, soziolinguistische und historische Aspekte eingegangen. Ein weiterer, ebenso häufiger Annäherungsversuch an das Feld Sprichwort erfolgt so, dass das Sprichwort im Unterschied zu ähnlichen, aber nicht gleichen sprachlichen Erscheinungen wie bspw. Sentenzen, geflügelten Worten, Aphorismen, Maximen und Slogans beschrieben wird oder darüber hinaus noch der Unterschied zwischen Sprichwort und sprichwörtlicher Redensart (vgl. Burger, 2012), zwischen Sprichwort und Idiom herausgearbeitet wird (Mieder, 1999). 
All diesen „definitorischen Spitzfindigkeiten“ zum Trotz erweisen sich diese Versuche der Grenzziehung als vage und fließend (Mieder, 1999:5). Da der Untersuchungsgegenstand dieses Beitrags die Sprichwörter sind, die bereits im SprichWort-Projekt (2007 - 2010) als Sprichwörter identifiziert und als frequent ermittelt wurden, scheint es sinnvoll, von der Arbeitsdefinition des Projekts auszugehen und sie nicht weiter zu diskutieren. Die Datenbank des Projekts enthält 300 Einträge bzw. Sprichwörter (mit Deutsch als Ausgangssprache), die folgendermaßen definiert wurden:

Sprichwörter [werden] als propositionale Phraseme mit unikalem Charakter aufgefasst, die also semantisch und syntaktisch den phraseologischen Kriterien der Idiomatizität und Stabilität entsprechen, meist eine metaphorische Natur haben und auf tradierte menschliche und kulturelle Erfahrungen hinweisen. Sie werden als „,in sich geschlossene Sätze, die durch kein lexikalisches Element an den Kontext angeschlossen werden müssen“ verstanden, die also ,als selbstständige ,Mikrotexte“ aufgefasst werden können“" (Burger, 2010:106, zit. nach Jesenšek, 2011:71).

Neben diesen explizit linguistisch orientierten Kriterien wurden im Projekt SprichWort noch die didaktische und inhaltliche Relevanz der ermittelten Einträge beachtet. Diese berücksichtigen den hohen Grad der Aktualität im gegenwärtigen Sprachgebrauch sowie die inhaltliche Relevanz gemäß dem Gemeinsamen europäischen Referenzrahmen für Sprachen (vgl. Jesenšek, 2011).

Die auf der SprichWort-Plattform dargestellte Sammlung von 300 Sprichwörtern ist somit ein, was die Zahlen angeht, begrenztes Korpus, in das die Sprichwörter nach eindeutigen, im Voraus festgelegten Kriterien aufgenommen wurden, nachdem sie aufgrund einer qualitativen, kriteriengeleiteten Differenzierung aus ca. 2000 registrierten Sprichwörtern isoliert wurden (vgl. Durčo, 2005 u. a.). Das Korpus, das eine linguistische und didaktische Relevanz sowie Frequenz und Aktualität auszeichnen, hat somit einen wichtigen pragmatischen und didaktischen Wert und ist noch dazu eine wichtige Quelle für verschiedene Forschungen.

\section{Das Medium Wörterbuch}

Das Wörterbuch, ein Nachschlagewerk, eine Datensammlung mit äußerer Zugriffsstruktur, die sprachliche Angaben zu lexikalischen Einheiten wie Wörtern, Wendungen, Morphemen etc. oder zu Begriffen enthält (vgl. Engelberg, Lemnitzer, 2009: 6), ist ein wichtiges Medium, eine wichtige Quelle für verschiedene Benutzerprofile. Hinsichtlich der anvisierten Benutzer erfüllt das Wörterbuch verschiedene Funktionen: es wird zur Hin- und/oder Herübersetzung verwendet, zum Verstehen (Hallsteinsdóttir 2009), zum Produzieren (fremdsprachiger) Texte (Bergerová, 2011), zur Suche nach zielsprachlichen Äquivalenten, nach grammatischen Eigenschaften (fremd)sprachlicher Einheiten oder nach typischen oder weniger typischen Kollokationen... (vgl. Storrer, 2001). Im Wirkungsbereich „Wörterbuch“ kursieren - genauso wie beim Sprichwort - viele Begriffe, die nicht eindeutig aufgefasst werden können. Es gibt viele verschiedene Wörterbücher und die Forscher versuchen, sie nach den unterschiedlichsten Kriterien zu klassifizieren bzw. zu typisieren. Da der Untersuchungsgegenstand dieses Beitrags drei typologisch gesehen verschiedene Wörterbücher sind, wird im Folgenden kurz auf die verschiedenen Wörterbuchtypen eingegangen. 
Hinsichtlich des Präsentationsmediums unterscheidet man einerseits Printwörterbücher oder gedruckte Wörterbücher, die Papier als Datenträger verwenden (vgl. Engelberg, Lemnitzer, 2009:6), in Form eines Buches erscheinen und den Benutzern in materieller Form in den Bibliotheken, Buchhandlungen oder zu Hause zur Verfügung stehen. Ferner gibt es auch sog. Internetwörterbücher, d. h. das Printwörterbuch erscheint nicht auf Papier, sondern es wird, so wie es ist, in eine elektronische Fassung verwandelt. Andererseits existieren elektronische bzw. digitale Wörterbücher, die auf elektronischen Datenträgern, z. B. auf CD-ROM oder im WWW publiziert werden und den Benutzern zahlreiche, sehr verschiedenartige, allgemeine, spezialisierte oder personalisierte Verwendungsmöglichkeiten bieten. Jene Wörterbücher, die über das WWW zugänglich sind, werden auch Online-Wörterbücher genannt (vgl. Storrer, 2001).

Sowohl das eine wie auch das andere Medium hat seine Stärken und Schwächen, Online-Wörterbücher (im Weiteren wird dieser Begriff verwendet) liegen allerdings wegen ihrer Aktualität, Interaktivität, relativ einfacher Bedienbarkeit, permanenter Verfügbarkeit und der Möglichkeit der kollaborativen Arbeit stark im Trend. ${ }^{1}$

Die Bestimmung der Wörterbuchgrundtypen hat laut Engelberg/Lemnitzer (2009) eine lange lexikographische Tradition. Während Kühn (1989) die Wörterbuchtypen nach ihren dominierenden Benutzungsfunktionen klassifizierte, konzipierte Hausmann (1989) eine sog. phänomenologische Klassifikation, bei der die zentralen Klassifikationskriterien unmittelbar erkennbar sind. Konkret heißt das, dass es einsprachige und zweisprachige Wörterbücher gibt und man in jeder dieser Klassen Allgemeinwörterbücher von Spezialwörterbüchern unterscheidet. Die Spezialwörterbücher werden dann noch weiter nach verschiedenen Kriterien klassifiziert, wie etwa bezüglich der anvisierten Benutzergruppen, der Textsorte(n), bestimmter Informationstypen oder Lemmatypen usw. (vgl. Engelberg, Lemnitzer, 2009:20-21). Hausmanns typologischer Vorschlag ist auch für die vorliegende Untersuchung relevant, weil er unmittelbar erkennbare, strukturelle und inhaltliche Ähnlichkeiten von Wörterbuchtypen hervorhebt.

Der Gegenstand der vorliegenden Untersuchung sind die drei Wörterbücher DWDS (Digitales Wörterbuch der deutschen Sprache), Langenscheidt (e-Großwörterbuch Deutsch als Fremdsprache) und PONS (zweisprachiges deutsch-slowenisches Wörterbuch).

Mit Bezug auf Hausmann kann bisher zusammengefasst werden, dass DWDS ein einsprachiges Allgemeinwörterbuch ist, das im Internet zugänglich ist und insofern ein einsprachiges Online-Wörterbuch genannt werden kann. Es ist ein wichtiges Nachschlagewerk, das neben den Grammatiken für die deutsche Sprache einen elementaren Baustein des Sprachsystems darstellt. Das Hauptmerkmal dieses Wörterbuchtyps bei der Lemma-Auswahl ist die Orientierung an der Standardsprache, die semasiologische Anordnung und das Angebot des mehr oder weniger vollen Informationsprogramms zu den Lemmata einschließlich der Aufarbeitung polysemantischer Wörter, Angaben zu den Verwendungsbereichen, kontextbezogener Funktionsangaben sowie authentischer Textbeispiele. Das DWDS gehört nach Engelberg und Lemnitzer zu den wichtigsten neueren Vertretern dieses Wörterbuchtyps (2009: 25).

\footnotetext{
${ }^{1}$ Im Onlinelexikon LEO (2011) wird prognostiziert, dass dieses den schleichenden Niedergang der gedruckten Sprachlexika darstellt. Gedruckte Wörterbücher hätten weitgehend ausgedient, behauptet Hans Riethmayer, Chef und Gesellschafter von Leo. Bereits im Jahr 1992 gründete ein Münchner Student unter dem Namen „Link everything online“, kurz „Leo“, ein Sprachenportal und brachte damit die großen Wörterbuchverlage Langenscheidt und Klett in Bedrängnis (http://www.handelsblatt.com/unternehmen/it-medien/onlinelexikon-loescht-leo-das-gedruckte-woerterbuch-aus/4183382.html, abgerufen am 24. Februar 2014).
} 
Das zweite für die Analyse ausgewählte Wörterbuch Langenscheidt ist ein „maßgeschneidertes, benutzerorientiertes Wörterbuch“, das typologisch Lernerwörterbuch genannt wird (Engelberg, Lemnitzer, 2009: 27). Dieser Wörterbuchtyp nimmt eine Sonderstellung unter den Wörterbuchtypen ein, denn die Lernerwörterbücher sind speziell für Benutzer im Zweit- bzw. Fremdsprachenerwerb konzipiert. Konzeptionell gesehen richten sie sich nach den Anforderungen des Nicht-Muttersprachlers, genauer eines fortgeschrittenen Fremdsprachenlerners. Vom Umfang her entsprechen sie den einbändigen Standardwörterbüchern, unterscheiden sich von diesen aber dadurch, dass die Anzahl der Lemmata geringer ist, die Angaben zu den einzelnen Lemmata aber wegen des spezifischen Benutzerprofils umfangreicher sind und in der zu lernenden Sprache angeboten werden (vgl. Engelberg, Lemnitzer, 2009:27, 29). Lernerwörterbücher sind in der Regel einsprachig und somit universell, bzw. für Lerner mit beliebigen Muttersprachen konzipiert. Engelberg und Lemnitzer weisen darauf hin, dass die Lexikographie des Deutschen eine Reihe von Lernerwörterbüchern hervorbrachte, unter denen auch das Lernerwörterbuch von Langenscheidt erwähnt wird (2009:29).

$P O N S$ ist das dritte Wörterbuch, das bezüglich der Sprichwörter analysiert wurde. Es handelt sich um ein zwei- bzw. mehrsprachiges Online-Wörterbuch, das konkret für 18 Sprachpaare verfasst wurde. Die Analyse greift die Daten auf, die im zweisprachigen deutsch-slowenischen PONS Wörterbuch vorhanden sind. ${ }^{2}$ Das zweisprachige Wörterbuch fungiert als ein Medium, in dem für die Lemmata der Ausgangssprache anstatt der Bedeutungsdefinitionen, die für einsprachige Wörterbücher charakteristisch sind, Äquivalente in der Zielsprache angeboten werden, die die Funktion der lexikografischen Paraphrase übernehmen. Bei Äquivalenzlücken werden die Bedeutungen in der Zielsprache paraphrasiert. Die Zielsprache spielt bei der Strukturierung der Wörterbuchartikel eine wichtige Rolle.

Jedes der drei in die Untersuchung aufgenommenen Wörterbücher hat seine Besonderheiten. Allen gemeinsam ist die mediale Form: DWDS, Langenscheidt und PONS sind digitale bzw. Online-Wörterbücher. Sie unterscheiden sich, was die Orientierung an der Benutzergruppe und was die Lemmata-Anzahl und -Präsentation angeht. Trotz dieser Unterschiede bzw. gerade wegen dieser Unterschiede sind sie für eine parömiologisch ausgerichtete Recherche von großem Interesse.

\section{Analyse der präsenz der Sprichwörter in den WörterbüChern}

\subsection{Forschungsfragen}

Das Ziel der empirischen Untersuchung war, Daten $\mathrm{zu}$ erheben, um die folgenden Forschungsfragen zu beantworten:

- Wie ist die Häufigkeitsverteilung der 300 Sprichwörter aus dem SprichWort-Korpus in den Wörterbüchern DWDS, Langenscheidt und PONS?

\footnotetext{
${ }^{2}$ Es ist wichtig zu betonen, dass die PONS-Wörterbücher (www.pons.eu) Teile des PONS-Sprachenportals sind. Das Sprachenportal PONS (www.pons.eu) enthält neben zweisprachigen Wörterbüchern für z. Z. 18 Sprachpaare auch einen Vokabeltrainer. Auf seiner Webseite steht, dass es für den Einsatz an Schulen, Hochschulen oder Sprachschulen sowie für den Einsatz im Büro oder den privaten Gebrauch geeignet ist. Das deutsch-slowenische Online-Wörterbuch von PONS und das slowenisch-deutsche Online-Wörterbuch von PONS enthalten je 75000 Lemmata.
} 
- Gibt es Unterschiede in der Anzahl der Sprichwörter in den Wörterbüchern, abhängig von der Art des Wörterbuchs - allgemeines einsprachiges Wörterbuch, einsprachiges Lernerwörterbuch oder zweisprachiges Wörterbuch?

- Wie viele der Sprichwörter sind in allen drei Wörterbüchern, in zwei der Wörterbücher, in einem einzigen oder in keinem der Wörterbücher nachweisbar?

- Wie sieht die Frequenzverteilung der Sprichwörter in den verschiedenen Sprichwortgruppen A bis E (A - sehr frequente bis E - wenig frequente Sprichwörter) aus?

- Welche Sprichwörter kommen in bestimmten Sprichwortgruppen (A bis E) und gleichzeitig in den untersuchten Wörterbüchern vor?

\subsection{Forschungsmethodologie}

Zur Durchführung der Untersuchung wurde die deskriptive und kausal-nichtexperimentelle Methode verwendet. Die 300 Sprichwörter des Korpus der SprichWort-Plattform wurden anhand ihrer repräsentativen Lemmata in den drei für die Untersuchung ausgewählten Wörterbüchern gesucht, identifiziert und somit ihre Präsenz erfasst, analysiert und die so erhaltenen Ergebnisse interpretiert.

\section{Resultate del analyse}

\subsection{Vorkommen der Sprichwörter in den drei Wörterbüchern}

In einem ersten Schritt wurde untersucht, wie viele der 300 aufgrund einer Korpusanalyse ausgewählten Sprichwörter der SprichWort-Plattform in den drei untersuchten Wörterbüchern vorkommen. Dabei zeigte sich, dass im DaF-Lernerwörterbuch von Langenscheidt mit 102 Sprichwörtern nur gut ein Drittel (34 \%) der Sprichwörter verzeichnet sind. Im DWDS, das in erster Linie für Muttersprachler zusammengestellt wurde, findet man allerdings auch nur gut die Hälfte ( $\mathrm{f}=154$ oder $51,7 \%$ ) der Sprichwörter. Lediglich ein gutes Viertel der Sprichwörter, d. h. 27,3\% ( $\mathrm{f}=83)$, ist im zweisprachigen deutsch-slowenischen Wörterbuch von PONS nachweisbar.

Wie erwartet enthält das einsprachige deutsche Wörterbuch DWDS die meisten Sprichwörter, allerdings ist der dort eruierte Prozentsatz von 51,7 \% der 300 Sprichwörter unerwartet gering, insbesondere, wenn man davon ausgeht, dass das DWDS ein einsprachiges, allgemeines Wörterbuch ist, das an die Zielgruppe der Muttersprachler gerichtet ist. Der erwartet geringere Anteil an Sprichwörtern in den Wörterbüchern Langenscheidt und PONS kann auf den geringeren Umfang an Wörterbucheinträgen zurückgeführt werden. Im Fall von PONS muss der Fakt berücksichtigt werden, dass beide Sprachen gleichermaßen vertreten sind und somit auf jede der zwei Sprachen weniger Wörterbucheinträge entfallen. 


\subsection{Nennungen der Sprichwörter in drei, zwei, einem oder keinem Wörterbuch}

Die Daten zu den in die drei Wörterbücher aufgenommenen Sprichwörtern wurden noch genauer analysiert: Untersucht wurde, wie viele der Sprichwörter in allen drei oder in zwei Wörterbüchern und in welchen der möglichen Zweierkombinationen sie zu finden sind. Des Weiteren wurde untersucht, wie viele Sprichwörter nur in einem Wörterbuch vorkommen und wie groß der Anteil der einzelnen Wörterbücher dabei ist. Es wurde auch überprüft, wie viele von ihnen in keinem der untersuchten Wörterbücher vorkommen.

Eine genauere Analyse der Distributionsdaten ergibt, dass die 82 (27,3\%) der im Wörterbuch PONS festgestellten Sprichwörter nicht in allen drei Wörterbüchern zu finden sind, diese Zahl ist bedeutend niedriger und beträgt lediglich 13,33\% ( $\mathrm{f}=40)$. In zwei der drei untersuchten Wörterbücher sind insgesamt 22,67\% $(\mathrm{f}=68)$ der Sprichwörter verzeichnet. Der Anteil der Sprichwörter, die nur in jeweils einem der drei Wörterbücher vorkommen, ist mit insgesamt 27,33 \% aller Sprichwörter $(\mathrm{f}=82)$ etwas höher als bei den Sprichwörtern, die in zwei Wörterbüchern vorkommen.

Noch größer ist der Anteil der Sprichwörter, die in keinem der Wörterbücher zu finden sind, dieser beträgt etwas mehr als ein Drittel der 300 Sprichwörter $(36,67 \%$ oder $\mathrm{f}=110)$.

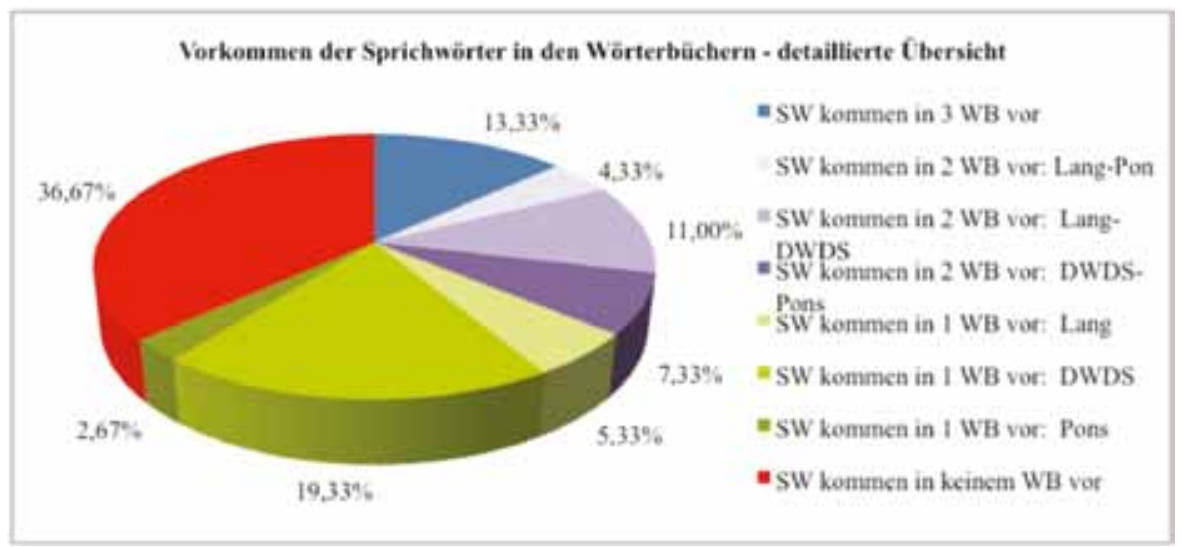

Diagramm 1: Detaillierte Aufschlüsselung der Nennungen der 300 Sprichwörter (SW) in den drei untersuchten Wörterbüchern

Eine detailliertere Aufschlüsselung der Daten zu der Sprichwörterpräsenz bedarf einer genaueren Interpretation: Bei der Hälfte der Sprichwörter $(f=150)$ ist das Resultat eindeutig, überraschend ist jedoch, dass davon ganze 36,67 \% $(f=110)$ auf Sprichwörter entfallen, die in keinem der drei untersuchten Wörterbücher vorhanden sind. Die restlichen 13,33\% dieser Hälfte $(f=40)$ sind in allen drei Wörterbüchern vorhanden, woraus geschlossen werden kann, dass die Wörterbuchautoren diesen Sprichwörtern eine große Bedeutung zumessen. Auf mögliche Gründe dafür wird später eingegangen.

Die Ergebnisse der Untersuchung zeigen weiter, dass 22,67 \% der Sprichwörter ( $\mathrm{f}=$ 68) in zwei Wörterbüchern vorkommen. Diese zu allgemeine Information muss aufgrund der verschiedenen Wörterbuchtypen und der Erwartungen, die diesen verschiedenen Typen 
entspringen, detaillierter untersucht werden. Die Aufschlüsselung der Ergebnisse der drei möglichen Wörterbuchkombinationen ergibt folgendes, teilweise unerwartetes Bild:

Die Frequenzanalyse zeigt, dass es die meisten Sprichwörter in der Wörterbuchkombination DWDS (einsprachiges allgemeines Wörterbuch) und Langenscheidt (einsprachiges Lernerwörterbuch) gibt, das sind $11 \%(f=33)$ der untersuchten 300 Sprichwörter. An zweiter Stelle ist mit 7,3\% $(\mathrm{f}=22)$ die Kombination DWDS und PONS (zweisprachiges Wörterbuch) zu finden, während die Kombination Langenscheidt und PONS lediglich 4,3 $\%(\mathrm{f}=13)$ gleiche Sprichwörter aufweist. Vor allem der letzte Prozentsatz ist überraschend gering, da erwartet wurde, dass zumindest in den zwei Wörterbüchern, deren Zielgruppe DaF-Lernende sind, eine grundlegende Auswahl für den Alltagsgebrauch bedeutender Sprichwörter zu finden sein müsste. Die erhaltenen Ergebnisse deuten dagegen darauf hin, dass die Sprichwörter eher arbiträr und unsystematisch in die einzelnen Wörterbücher - hier die Wörterbücher Langenscheidt und PONS - aufgenommen wurden.

Die Frequenzdaten bezüglich der Sprichwörter, die nur in einem der drei untersuchten Wörterbücher zu finden sind, stehen im Unterschied zu denen, die in zwei Wörterbüchern vorhanden sind, im Einklang mit den Wörterbuchtypen, denen sie angehören: So können $19,33 \%(\mathrm{f}=58)$ im DWDS, 5,33\% $(\mathrm{f}=16)$ im Langenscheidt und nur 2,67\% $(\mathrm{f}=8)$ im PONS identifiziert werden.

\subsection{Prozentueller Anteil der Nennungen der Sprichwörter innerhalb der Sprichwort- gruppen $(\mathbf{A}-\mathbf{E})$}

Die 300 Sprichwörter der SprichWort-Plattform wurden aufgrund einer Korpusanalyse bezüglich ihrer Häufigkeit rangiert (vgl. Kacjan, 2013). Dabei entstanden die Sprichwortgruppen A bis E, wobei in der Gruppe A die Sprichwörter zu finden sind, die im untersuchten Korpus (bei der Korpusanalyse wurden auch Varianten der Sprichwörter berücksichtigt) am häufigsten vorkommen, und in Gruppe E die Sprichwörter mit der geringsten festgestellten Häufigkeit ${ }^{3}$. Wenn die Sprichwortnennungen in den digitalen Wörterbüchern zu dem jeweiligen Umfang der Sprichwortgruppen in Beziehung gesetzt werden, ergibt sich folgendes Bild:

Bei den Sprichwörtern mit Nennungen in allen drei digitalen Wörterbüchern zeigt sich die Tendenz, dass mit fallender Korpusfrequenz auch der prozentuelle Anteil der dreifachen Belege von $39,1 \%$ in Sprichwortgruppe A bis auf $0 \%$ in Sprichwortgruppe E verringert. Eine ähnliche Tendenz ist auch bei den zweifachen und einfachen Nennungen zu verzeichnen, wenn auch mit niedrigeren Ausgangsprozenten (2 Nennungen beginnt mit 26,1\% und eine Nennung mit $13 \%$ ) und dem gleichen Ergebnis in Sprichwortgruppe E (jeweils 0\%).

Diametral entgegengesetzt entwickelt sich die Situation bei den Sprichwörtern, die in keinem der drei digitalen Wörterbücher vorkommen: Von den 21,7 \% in der Sprichwortgruppe A und einem leicht gefallenen Prozentsatz von $19 \%$ in der Sprichwortgruppe B steigt der

\footnotetext{
${ }^{3}$ Parallel zur beschriebenen Korpusanalyse wurde die Frequenz der 300 Sprichwörter der SprichWort-Plattform im Internetbrowser Google überprüft, der von Fachleuten als einer der besten bzw. genauesten Suchmaschinen beschrieben wird. Die dort erhaltenen Daten zur Trefferanzahl können als im Alltag aktuell bezeichnet werden. Die so erhaltenen Ergebnisse zeigten eine starke Kongruenz mit den Daten der Korpusanalyse, die die Relevanz und Häufigkeit der vorgeschlagenen Sprichwortgruppen (A bis E) bestätigten und aus diesem Grunde in die dem Beitrag zugrunde liegende Untersuchung aufgenommen wurden.
} 
Prozentsatz auf 32,5\% in Sprichwortgruppe C, weiter auf 52,9 \% in Sprichwortgruppe D und schließlich auf ganze $100 \%$ in der Sprichwortgruppe E, in der nur Sprichwörter ohne Wörterbuchnennungen zu finden sind. Während die Frequenz der Sprichwörter geringer wird, steigt der Prozentsatz derer, die in keinem der Wörterbücher zu finden sind, konkret von ca. einem Fünftel der Sprichwörter in den beiden frequentesten Sprichwortgruppen bis auf 100 $\%$ in der am wenigsten frequenten Sprichwortgruppe E.

Aus den bisherigen Daten kann die Schlussfolgerung gezogen werden, dass - wie erwartet - Sprichwörter, die im Korpus häufiger vorkommen, auch mit größerer Wahrscheinlichkeit und Frequenz in den Wörterbüchern nachgewiesen werden können, auch wenn diese zu drei sehr unterschiedlichen Wörterbuchtypen gehören. Diese auf den ersten Blick eindeutigen Aussagen bedürfen allerdings weiterer Analyse und Differenzierung. Diese Schlussfolgerung gilt im vollen Ausmaß für die Sprichwörter, die in allen drei Wörterbüchern zu finden sind: Je häufiger die Sprichwörter im Korpus anzutreffen sind, desto häufiger kommen sie auch in allen drei Wörterbüchern vor.

Ein ähnliches Bild ergibt sich bei den Sprichwörtern, die in zwei der drei Wörterbücher verzeichnet sind:

In Diagramm 2 zeigt die obere Linie das Gesamtbild, das bei den Sprichwörtern zu erkennen ist, die in jeweils zwei der drei Wörterbildern vorkommen.

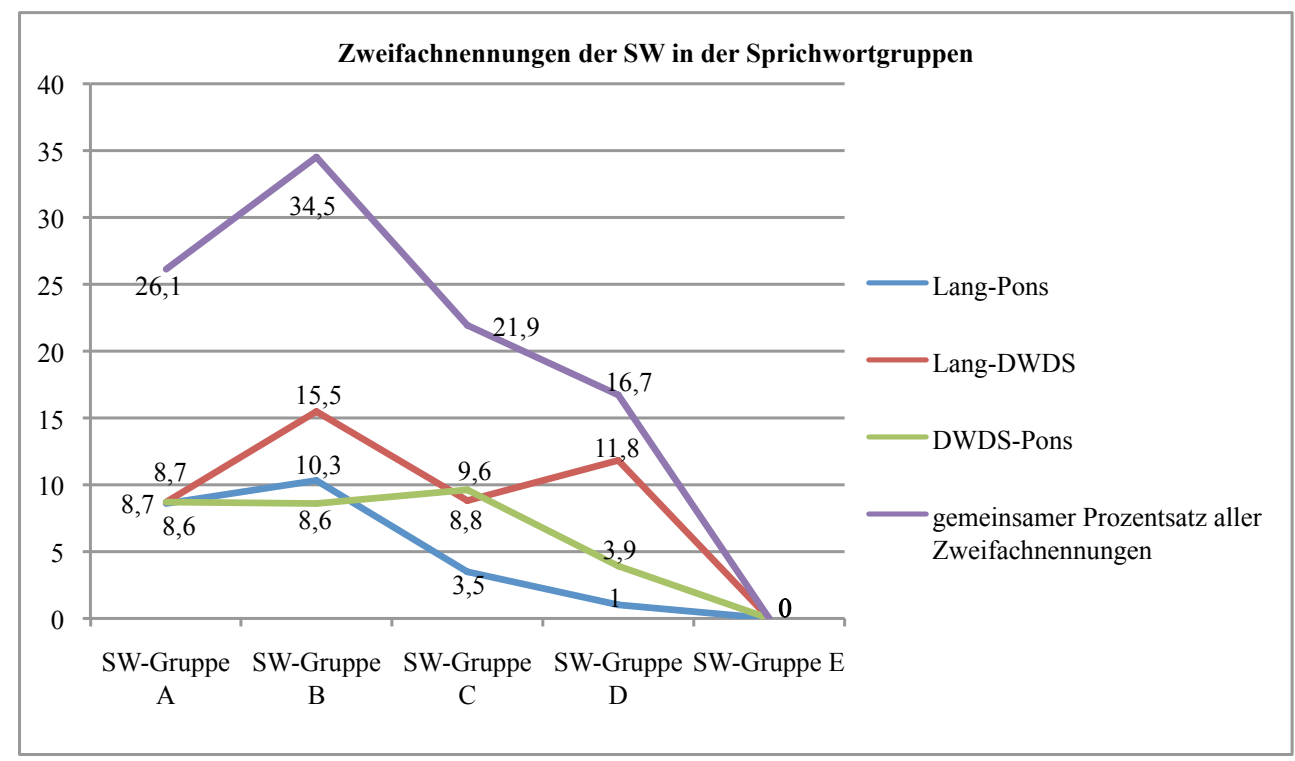

Diagramm 2: Zweifachnennungen der SW innerhalb der Sprichwortgruppen

Erwartet gering ist die Anzahl der Sprichwörter, die in dem einsprachigen DaF-Lernerwörterbuch Langenscheidt und gleichzeitig im zweisprachigen Wörterbuch PONS vorkommen. Die beiden Wörterbuchkombinationen Langenscheidt + DWDS sowie DWDS + PONS entsprechen ebenso den Erwartungen: Der prozentuelle Anteil der verzeichneten Sprichwörter 
in den beiden einsprachigen Wörterbüchern $($ Lang $+D W D S)$ ist etwas größer und leicht anders verteilt als in der Kombination ein- und zweisprachiges Wörterbuch (DWDS + PONS).

Die prozentuelle Verteilung der Sprichwörter, die in nur jeweils einem Wörterbuch vorkommen, sieht folgendermaßen aus:

In Diagramm 3 ist mit der obersten Linie der prozentuelle Anteil der Sprichwörter angegeben, die nur in einem der drei untersuchten Wörterbücher vorzufinden sind. Eine genauere Aufschlüsselung der Ergebnisse für jedes einzelne der drei Wörterbücher ergibt keine unerwarteten Abweichungen: Das zweisprachige Wörterbuch von PONS enthält weniger einmalig genannte Sprichwörter als die beiden einsprachigen, wobei es aber nur leicht hinter Langenscheidt zurückliegt. Beide liegen in allen Sprichwortgruppen weit unter $10 \%$. Ganz anders sieht es beim DWDS aus: Dort ist der prozentuelle Anteil der einmalig genannten Sprichwörter im Vergleich zu den beiden anderen größer und liegt in allen Sprichwortgruppen mit Ausnahme der Sprichwortgruppe E zwischen $13 \%$ (Sprichwortgruppe A) und 32,5\% (Sprichwortgruppe C).

Während die Kurve bei den Sprichwörtern, die in zwei der drei Wörterbücher vorkommen (vgl. Diagramm 2), rechts fallend ist, weisen die Kurven der Sprichwörter, die in nur einem Wörterbuch zu finden sind, eher eine Normalverteilung auf, wobei DWDS eher rechtslastig und $P O N S$ eher linkslastig ist, also im $P O N S$ verhältnismäßig mehr frequentere Sprichwörter vorkommen während im $D W D S$ die weniger frequenten Sprichwörter verhältnismäßig zahlreicher vertreten sind.

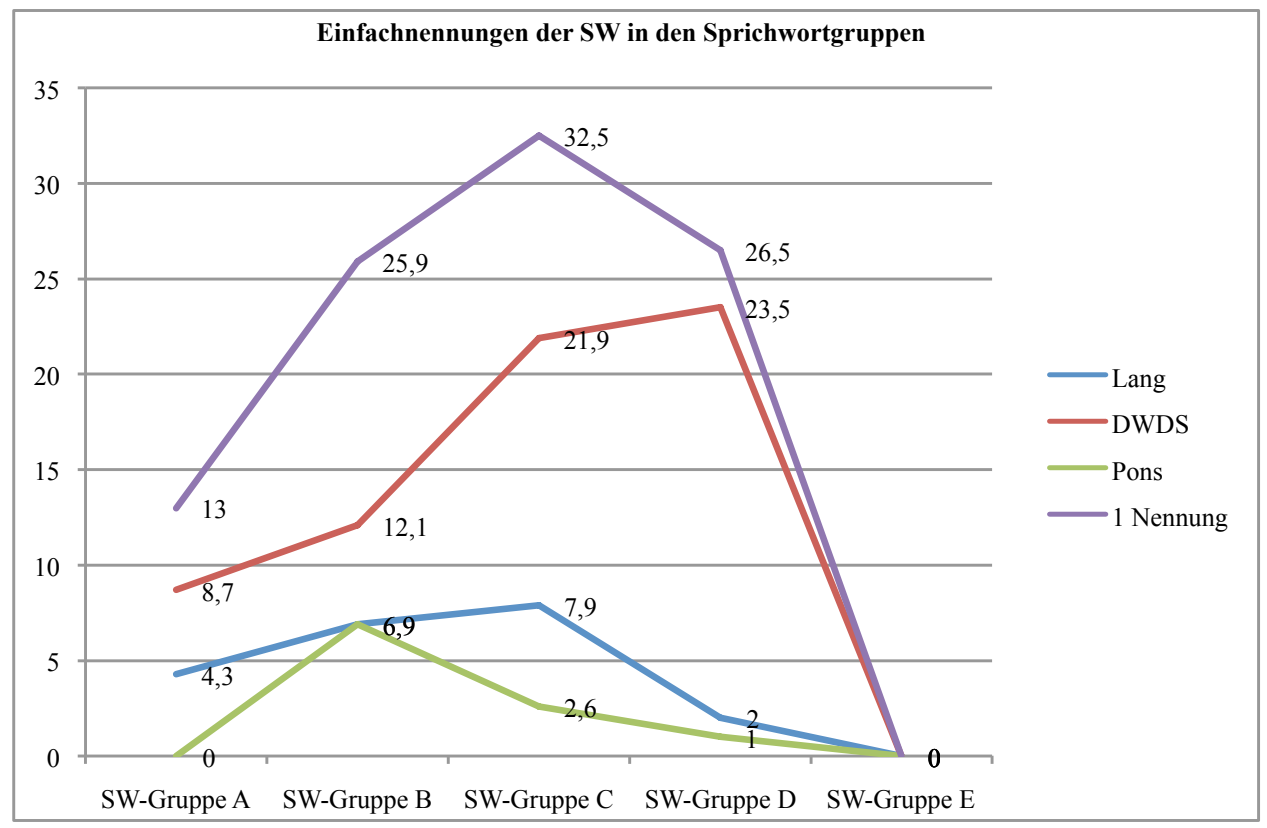

Diagramm 3: Einfachnennung der SW innerhalb der Sprichwortgruppen 


\subsection{Exkurs: Sprichwörter konkret}

In den vorangegangen Abschnitten ging es um die Frequenz und prozentuelle Distribution der Sprichwörter, die so gewonnenen Analyseergebnisse wurden auch mit Diagrammen veranschaulicht. Im Weiteren werden die Statistiken mit konkreten Sprichwörtern illustriert. Dabei geht es um die Sprichwörter, die in allen drei Wörterbüchern verzeichnet sind und der frequentesten Sprichwortgruppe (A) angehören. So werden die bisher präsentierten quantitativen Angaben zu den Sprichwörtern erweitert und es wird dabei versucht, einen eventuell gemeinsamen Nenner der Sprichwörter in den einzelnen Gruppen - bspw. gemeinsame Themenfelder, vergleichbare morphologische Eigenschaften usw. - zu eruieren.

Wie bereits mehrfach festgestellt wurde, sind lediglich 40 der 300 Sprichwörter der SprichWort-Plattform in allen drei untersuchten Wörterbüchern verzeichnet. Davon zählen $22,5 \%$ der Sprichwörter $(f=9)$, die in allen drei Wörterbüchern angeführt werden, zur ersten, frequentesten Sprichwortgruppe A:

Der Schein trügt; Der Zweck heiligt die Mittel; Ausnahmen bestätigen die Regel; Aller Anfang ist schwer; Viel Lärm um nichts; Es ist nicht alles Gold, was glänzt; Kleider machen Leute; Liebe geht durch den Magen; Zeit ist Geld.

Weitere $30 \%$ der Sprichwörter ( $\mathrm{f}=12$ ) gehören zur zweitfrequentesten Sprichwortgruppe B:

Aus den Augen, aus dem Sinn; Übung macht den Meister; Viele Köche verderben den Brei; Andere Länder, andere Sitten; Kleinvieh macht auch Mist; wer zuerst kommt, mahlt zuerst; Eine Schwalbe macht noch keinen Sommer; Hochmut kommt vor dem Fall; Irren ist menschlich; Der Apfel fällt nicht weit vom Stamm; Ohne Fleiß kein Preis; Aus Schaden wird man klug.

$37,5 \%$ der Sprichwörter ( $f=15$ ) zählen zu den mittelfrequenten Sprichwörtern der Sprichwortgruppe C:

Noch ist nicht aller Tage Abend; Jeder ist seines Glückes Schmied; Wer A sagt, muss auch B sagen; Lügen haben kurze Beine; Wer wagt, gewinnt; Ein Unglück kommt selten allein; Probieren geht über Studieren; Wer nicht hören will, muss fühlen; Wer zuletzt lacht, lacht am besten; Lieber den Spatz in der Hand als die Taube auf dem Dach; Hunde, die bellen, beißen nicht; Was sich liebt, das neckt sich; Eigenlob stinkt; Die Katze lässt das Mausen nicht; Gebranntes Kind scheut das Feuer.

$10 \%$ der Sprichwörter $(\mathrm{f}=4)$ sind der weniger frequenten Sprichwortgruppe D zuzuordnen:

Unkraut vergeht nicht; Müßiggang ist aller Laster Anfang; Vorsicht ist besser als Nachsicht; Frisch gewagt ist halb gewonnen.

In der am wenigsten frequenten Sprichwortgruppe F sind keine Sprichwörter verzeichnet, die in allen drei Wörterbüchern anzutreffen wären.

Das Ziel, mit dem Auflisten der 40 Sprichwörter, die in allen drei Wörterbüchern verzeichnet sind, einen gemeinsamen Nenner der Sprichwörter in den einzelnen Sprichwortgruppen zu finden, ist gescheitert. Die Sprichwörter in den einzelnen Sprichwortgruppen sind so unterschiedlich, dass es weder kontextuelle, semantische oder morphologische noch irgendwelche andere Gemeinsamkeiten innerhalb der Sprichwortgruppen gibt. Es konnten 
auch keine bedeutenden Unterschiede zwischen den Sprichwortgruppen identifiziert werden. Repräsentative diesbezügliche Daten könnten eventuell anhand einer soziolinguistischen Analyse gefunden werden, in der auch die Wörterbuchverwender und/oder die Wörterbuchautoren berücksichtigt würden.

\section{Fazit und Ausblick}

Online-Wörterbücher sind ein wichtiges Medium für jeden (Fremd-)Sprachenunterricht. Sie weisen bestimmte Qualitäten auf, sind für Benutzer sehr praktisch und für Autorenteams sowie Forscher verschiedener Forschungsdisziplinen aktuell und interessant. So war es das Ziel der vorliegenden lexikographisch orientierten Analyse, unterschiedliche Typen von OnlineWörterbüchern auf die Präsenz eines kleinen, aber umso wichtigeren Segments jeder Sprache, d. h. die Sprichwörter hin zu untersuchen. Dabei ging es sowohl um bewusst ausgewählte unterschiedliche Typen von Online-Wörterbüchern als auch um ein ganz bestimmtes Korpus von aktuellen, relevanten und frequenten Sprichwörtern. Diese Analysevorgaben sind wichtig, damit aus den Ergebnissen zumindest eine allgemeine Tendenz abgeleitet werden kann, die nicht nur speziell für die ausgewählten Wörterbücher und Sprichwörter im DaF relevant ist.

Bei den untersuchten digitalen Wörterbüchern handelt es sich um drei wichtige Typen von Online-Wörterbüchern, die im Prinzip jede Sprache hat: ein einsprachiges allgemeines Wörterbuch (hier: das einsprachige allgemeine deutsche Wörterbuch $D W D S$ ), ein einsprachiges Lernerwörterbücher (hier Langenscheidt) und ein zweisprachiges deutsch-slowenisches Wörterbuch (hier: PONS). Zusammenfassend kann festgehalten werden, dass im Rahmen der Analyse eigentlich viele der Sprichwörter in den Wörterbüchern festgestellt werden konnten, wobei allerdings in den verschiedenen Wörterbüchern unterschiedliche und unterschiedlich viele Sprichwörter vorhanden sind. Das Problem ist, dass in den Wörterbüchern die Kriterien für die Aufnahme (oder Nicht-Aufnahme) eines Sprichworts in das jeweilige Wörterbuch nicht angeführt sind und sie somit auch nicht wiedergegeben werden können. Unter Berücksichtigung der Wörterbuchtypen kann man spontan, aber auch aufgrund der aufgestellten Hypothese schlussfolgern, dass es in dem einsprachigen allgemeinen Wörterbuch (in der vorliegenden Untersuchung DWDS), das sich in erster Linie an Muttersprachler richtet, viele bzw. die meisten der frequenten, aktuellen und für eine Sprache relevanten Sprichwörter gibt. Die Analyse ergab allerdings auch ein etwas anderes Bild: Im DWDS gibt es bspw. im Verhältnis zum Gesamtumfang des Wörterbuchs weniger Sprichwörter (der 300 Sprichwörter der SprichWort-Plattform) als im zweisprachigen Wörterbuch PONS. Hierbei stellt sich die Frage, ob man bei der Analyse von anderen sprachlichen Einheiten in diesem Wörterbuchtyp auch zu solchen Ergebnissen kommen würde? Oder sind andere sprachlichen Einheiten im Wörterbuch besser vertreten? Wenn ja, wie stark und welche Einheiten sind das? Interessante und wichtige Fragen, die die Grundlage weiterer Untersuchungen und Analysen sein könn(t)en.

Aus der festgestellten Präsenz der Sprichwörter in den Wörterbüchern wurde dann in einem weiteren Schritt versucht, ein System oder Prinzip abzuleiten, nach dem die Sprichwörter Eingang in die verschiedenen Wörterbuchtypen gefunden haben oder davon ausgeschlossen wurden. In diesem Kontext zeigte die Untersuchung, in der überprüft wurde, ob die Sprichwörter in allen drei, in zwei in einem oder in keinem Wörterbuch präsent 
sind, sehr variable und unsystematische Resultate. Auch der vorgenommene Vergleich der Analyseergebnisse mit Daten einer Untersuchung, in der Kacjan (2013) die Häufigkeit der Sprichwörter im SprichWort-Korpus mit ihrer Häufigkeit in der Suchmaschine Google abglich und zu sehr ähnlichen Resultaten kam, führte zu der Feststellung, die Integration von Sprichwörtern in die Wörterbücher sei unsystematisch und unübersichtlich. Dies wird auch in der Analyse der Liste der Sprichwörter der ersten, frequentesten Sprichwortgruppe (A) bestätigt, in der keine kontextuellen, morphologischen, thematischen oder anderen gemeinsamen Charakteristiken festgestellt werden konnten, aufgrund derer die Sprichwörter in die Wörterbücher aufgenommen wurden.

Die empirische Untersuchung bestätigte ein weiteres Mal, dass die Erstellung eines Wörterbuchs ein sehr gewagtes Unterfangen ist. Das flexible, komplizierte und komplexe Sprachsystem in eine feste Form, konkret hier in die Form eines Wörterbuchs zu fassen, unterliegt immer bestimmten Restriktionen. Es gibt solche, die bewusst in Kauf genommen werden, aber auch solche, die bei dieser Fülle an zu berücksichtigenden Aspekten unbewusst ausgelassen werden. Das größte Problem, das bei der Arbeit mit sprachlichen Einheiten, in diesem Fall mit Sprichwörtern aufscheint, ist der Eindruck, bei der Auswahl der Sprichwörter für einen der Wörterbuchtypen seien bei den Wörterbuchverfassern und -verlegern die Intuition, Spontaneität sowie ein eingeschränktes lexikographisches Wissen und ein niedriges Sprachbewusstsein dominant. Unbedingt notwendig wären, ganz im Gegenteil, eine methodologische und inhaltliche Systematik, ausreichendes lexikographisches Wissen, im Voraus gestellte und gut durchdachte Kriterien für die Integration von möglichst vielen sprachlichen Einheiten in die Wörterbücher, darunter auch Sprichwörter, ein sehr großes Maß an sprachlicher Sensibilität sowie ein stark ausgebildetes Sprachbewusstsein aller Beteiligten. Wie bereits der Titel des Beitrags andeutet, muss abschließend festgestellt werden, dass die Präsenz der Sprichwörter in den Wörterbüchern für $\mathrm{DaF}$ und vermutlich auch für andere (Fremd)Sprachen nicht optimal ist, sondern ein noch zu realisierendes Potential hat, womit man sich wieder bei dem in der Einleitung erwähnten Sprichwort „Was nicht ist, kann ja noch werden“" wiederfindet.

\section{LITERATURVERZEICHNIS}

Bergerová, H. (2011). »Lernerlexikographie und Fremdsprachenerwerb. Desiderata und Perspektiven am Beispiel phraseologischer Lernerwörterbücher.«, in Aussiger Beiträge, 5: 147-161.

Burger, H. (2012). »Sprichwort und Redensart: Gemeinsamkeiten und Unterschiede - theoretisch und textuell, synchron und diachron betrachtet«, in K. Steyer (Hrsg.), Sprichwörter multilingual. Tübingen: Narr, 45-78.

Durčo, P. (2005). Sprichwörter in der Gegenwartssprache. Trnava: UCM.

Engelberg, S. und Lemnitzer, L. (2009). Lexikographie und Wörterbuchbenutzung. Tübingen: Stauffenburg.

Europarat (2001). Gemeinsamer europäischer Referenzrahmen für Sprachen: lernen, lehren, beurteilen. Berlin, München: Langenscheidt.

Hallsteinsdóttir, E. (2009). »Zweisprachige Lernerphraseographie aus funktionaler Sicht.«, in C. Mellado Blanco (Hrsg.), Theorie und Praxis der idiomatischen Wörterbücher. Berlin: de Gruyter. Lexicographica. Series Maior, 203-226. 
Jesenšek, V. (2011). »Sprichwörter im Wörterbuch.«, in Linguistik online, 47, 3: 67-78.

Kacjan, B. (2013). »Sprichwörter zwischen korpusbasierter Frequenzanalyse und DaF-Wörterbüchern«, in C. Konecny et. al. (Hrsg.), Phraseologie im Sprachunterricht und in der Sprachendidaktik. Maribor: Mednarodna založba Oddelka za slovanske jezike in književnosti, FF, 71-87.

Kispál, T. (2007). »Sprichwörtersammlungen«, in H. Burger und D. Dobrovol'skij und P. Kühn und N. R. Norrick (Hrsg.), Phraseologie/Phraseology. Ein internationales Handbuch zeitgenössischer Forschung/ An International Handbook of Contemporary Research. Berlin: de Gruyter, 414-422.

Kühn, P. (1989). »Typologie der Wörterbücher nach Benutzungsmöglichkeiten«, in F.J. Hausmann et al. (Hrsg.), Wörterbücher. Ein internationales Handbuch zur Lexikographie. Berlin, New York: De Gruyter, 111-127.

Mieder, W. (1999). »Sprichwörter in den größeren allgemeinen und phraseologischen Wörterbüchern Deutsch-Englisch/Englisch-Deutsch«, in H.E. Wiegand (Hrsg.), Studien zur zweisprachigen Lexikographie mit Deutsch IV. Hildesheim, New York: Olms, 1-40.

Mieder, W. (2010). Sprichwörter/Redensarten. Sprichwörter/Redensarten - Parömiologie. Band 27. Heidelberg: Julius Groos.

Steyer, K. (Hrsg.) (2012). Sprichwörter multilingual. Theoretische, empirische und angewandte Aspekte der modernen Parömiologie. Tübingen: Narr.

Storrer, A. (2001). »Digitale Wörterbücher als Hypertexte: Zur Nutzung des Hypertextkonzepts in der Lexikographie«, in I. Lemberg und B. Schröder und A. Storrer, (Hrsg.), Chancen und Perspektiven computergestützter Lexikographie. Hypertext, Internet und SGML/XML für die Produktion und Publikation digitaler Wörterbücher. Tübingen: Niemeyer, 88-104.

Ulčnik, N. (2011). »Pregovori v procesu spreminjanja« [Sprichwörter im Prozess der Veränderung], in B. Krakar Vogel (Hrsg.), Slavistika v regijah - Maribor. Ljubljana: Zveza društev Slavistično društvo Slovenije, 63-68.

Wiegand, H. E. et. al. (Hrsg.) (1989). Wörterbücher. Ein internationales Handbuch zur Lexikographie, 1. Teilband. Berlin, New York: de Gruyter.

http://www.dwds.de/, letzter Zugriff 24. Februar, 2014.

http://www.handelsblatt.com/unternehmen/it-medien/onlinelexikon-loescht-leo-das-gedrucktewoerterbuch-aus/4183382.html, letzter Zugriff 24. Februar, 2014.

http://de.pons.eu/, letzter Zugriff 24. Februar, 2014.

http://www.sprichwort-plattform.org/sp/Projekt, letzter Zugriff 24. Februar, 2014.

http://www.woerterbuch.langenscheidt.de/, letzter Zugriff 24. Februar, 2014. 